Document downloaded from:

http://hdl.handle.net/10251/99373

This paper must be cited as:

Cutanda-Henriquez, V.; Andersen, PR.; Jensen, JS.; Juhl, PM.; Sánchez-Dehesa MorenoCid, J. (2017). A Numerical Model of an Acoustic Metamaterial Using the Boundary Element Method Including Viscous and Thermal Losses. Journal of Computational Acoustics. 25(4):1750006-1-1750006-11. doi:10.1142/S0218396X17500060

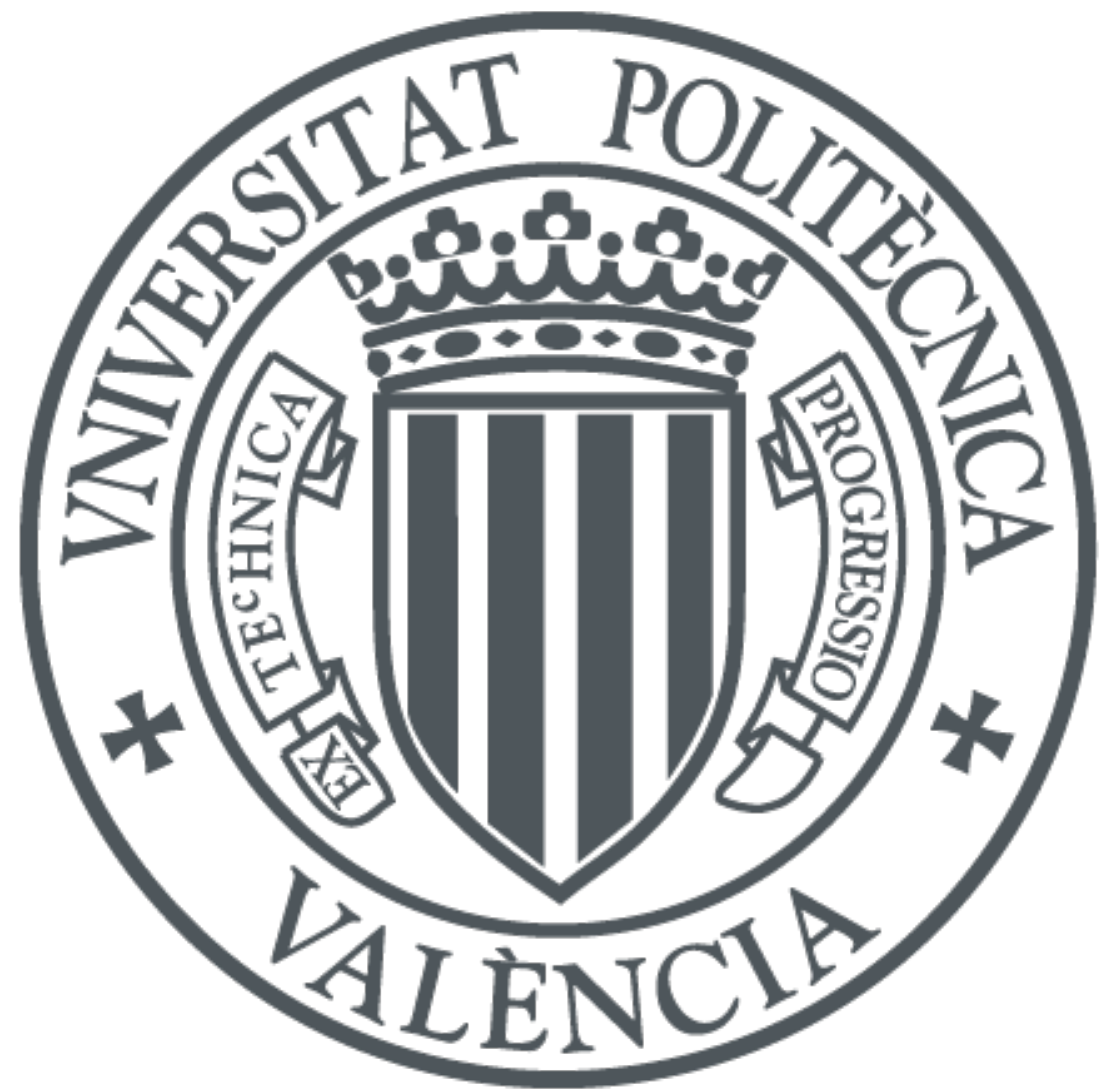

The final publication is available at

https://doi.org/10.1142/S0218396X17500060

Copyright World Scientific

Additional Information 


\title{
A Numerical Model of an Acoustic Metamaterial using the Boundary Element Method Including Viscous and Thermal Losses
}

\author{
V. Cutanda Henríquez*, P. Risby Andersen ${ }^{\dagger}$, J. Søndergaard Jensen ${ }^{\ddagger}$ \\ Centre for Acoustic-Mechanical Micro Systems, Technical University of Denmark \\ Ørsteds Plads, Building 352, DK-2800, Kgs. Lyngby, Denmark \\ *vcuhe@elektro.dtu.dk \\ ${ }^{\dagger}$ prand@elektro.dtu.dk \\ ${ }_{\ddagger}^{\ddagger}$ json@elektro.dtu.dk \\ P. Møller Juhl \\ Marsk Mc-Kinney Møller Institute, University of Southern Denmark, \\ Campusvej 55, DK-5230 Odense M, Denmark \\ pmjuhl@mmmi.sdu.dk \\ J. Sánchez-Dehesa \\ Wave Phenomena Group, Department of Electronic Engineering, Universitat Politècnica de València, \\ Camino de Vera s.n. (building 7F), ES-46022 Valencia, Spain \\ jsanched@upv.es \\ Received (Day Month Year) \\ Revised (Day Month Year)
}

\begin{abstract}
In recent years, Boundary Element Method (BEM) and Finite Element Method (FEM) implementations of acoustics in fluids with viscous and thermal losses have been developed. They are based on the linearized Navier-Stokes equations with no flow. In this paper, such models with acoustic losses are applied to an acoustic metamaterial. Metamaterials are structures formed by smaller, usually periodic, units showing remarkable physical properties when observed as a whole. Acoustic losses are relevant in metamaterials in the millimeter scale. In addition, their geometry is intricate and challenging for numerical implementation. The results are compared with existing measurements.
\end{abstract}

Keywords: boundary element method; acoustic metamaterials; viscous and thermal acoustic losses

\section{Introduction}

Acoustic metamaterials are artificial periodic structures with unit cells containing features such as resonators or scatterers. ${ }^{1}$ The macroscopic acoustic properties may show values that are outside the limits of what is achievable with regular materials in the sub-wavelength range, leading to interesting applications. Acoustic metamaterials have been studied with approximate analytical models and numerical methods, and measurements have been carried out. $^{2}$

Sound waves are subject to losses due to fluid viscosity and heat exchange. Such losses are particularly relevant in the vicinity of boundaries: On one hand, particle velocity must 
match boundary velocity in its normal and tangential components (non-slip condition), creating a viscous boundary layer where viscous losses are relevant. On the other hand, the boundary usually has a much higher thermal conductivity than the fluid, thus reducing the temperature variations associated with acoustic waves, generating losses in a thermal boundary layer of a similar thickness, which for audible frequencies in air varies from a fraction of a millimeter to a few micrometers. These viscous and thermal losses are usually taken into account as an acoustic impedance of the boundary, and the sound field can be described by the lossless wave equation. ${ }^{3}$ However, when the dimensions of the domain or some part of it are similar to the boundary layer thickness, it becomes necessary to take losses in the domain into account. This is the case of small acoustic devices such as acoustic transducers, couplers, hearing aids and small-scale acoustic metamaterials.

Recent research has led to new implementations of viscous and thermal losses in numerical methods. The Boundary Element Method (BEM) is the basis of a full implementation with acoustic losses. ${ }^{4,5}$ It is based on the Kirchhoff decomposition of the Navier-Stokes equations, where the so-called viscous, thermal and acoustic modes are described with different equations and coupled at the boundaries. ${ }^{3,6,7}$ As in the lossless case, BEM meshes are defined on the boundaries, where the three modes are coupled through boundary conditions. The BEM with losses has been implemented for research purposes with the objective of dealing with particularly challenging and computationally demanding cases, such as condenser microphones and the structure brought forward in this paper. ${ }^{8,9}$

The Finite Element Method (FEM) can also be used to directly solve the no-flow linearized Navier-Stokes equations with no further hypotheses, and is suitable for any geometry. ${ }^{10,11,12,13}$ The FEM implementation with losses is available with some commercial FEM software packages. ${ }^{14}$

Besides the full BEM and FEM with viscous and thermal losses, there are other implementations where some restricting assumptions are made. ${ }^{15,16}$ These alternative implementations are not investigated in this paper.

In a recent publication an acoustic metamaterial was studied using theoretical models, complemented with experiments. ${ }^{17}$ However, poor agreement was found, and the authors speculated that the possible cause was the fact that no losses were included in the theoretical models. In this paper, BEM and FEM models with viscous and thermal losses are presented and employed for modeling the metamaterial. This particular problem has three features, that makes it relevant as a test case: i) it has implications that are relevant for metamaterial research where acoustics losses are often neglected, ii) it is a suitable problem where implementations of losses need to handle an intricate geometry, and iii) it is on the limit of what is possible with numerical tools in terms of computational load. The present paper is motivated by issues ii) and iii). There are indeed in the literature some examples of computationally heavy models and also containing intricacies. ${ }^{15,18}$ Acoustic metamaterials, however, must contain and are based on internal, often complex, periodic structures where losses in individual units add up in the complete setup. They are a class of problems where full numerical models with no geometrical restrictions such as the aforementioned full BEM and FEM implementations with losses are the obvious choice. 
In section two, the acoustic metamaterial and its desired properties are described, outlining the relevant background of the investigation in [17]. Section three contains descriptions of the BEM and FEM models with losses employed. The results of the simulation with losses are presented and compared with existing measurements in section four. The paper is summarized in the conclusions section.

\section{The acoustic metamaterial}

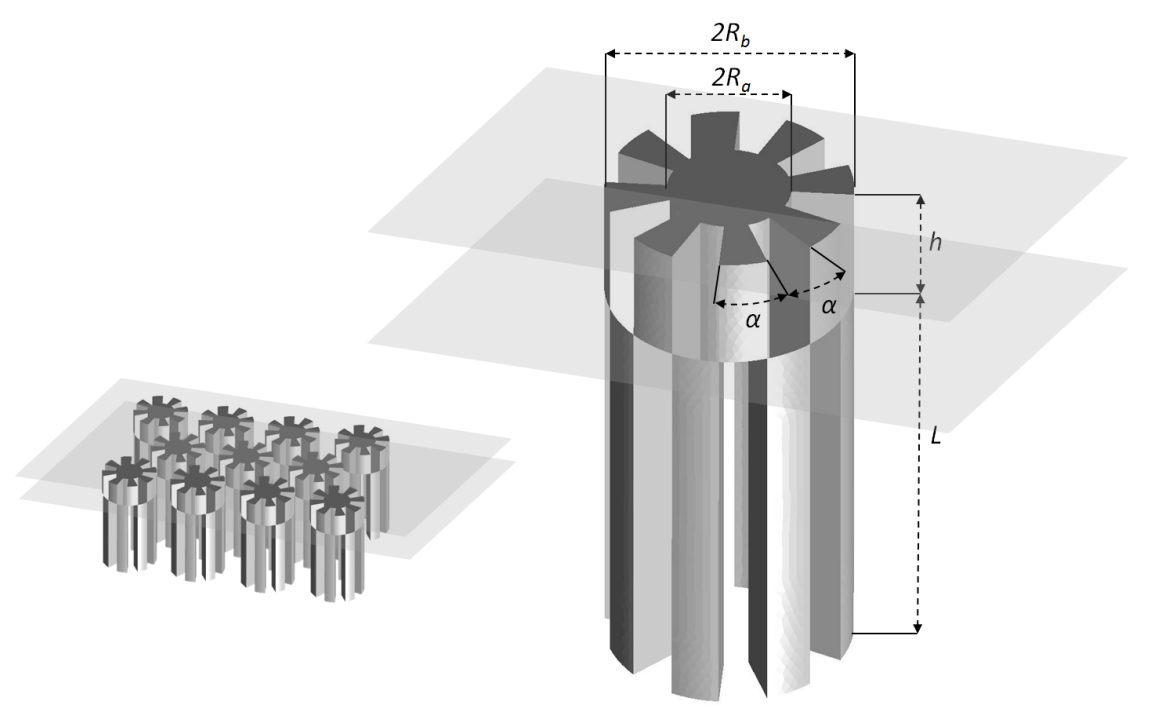

Fig. 1. Metamaterial single unit as described by Graciá-Salgado et al., with the relevant dimensions. The structure is embedded between two horizontal planes separated by distance $h$, leaving the lower part of length $L$ below the lower plane and forming wells that may act as resonant elements. $R_{a}$ is the inner radius, $R_{b}$ is the outer radius and $\alpha$ is the angle that corresponds to a well (or between wells) sector. The units are arranged regularly as a grid to form the metamaterial, as shown in the sketch to the left.

An acoustic metamaterial is presented and studied in Ref. [17] using analytical and numerical models that do not include losses. This material is formed by unit cells as shown in Fig. 1, which are arranged in a periodic structure embedded between two horizontal planes separated by the distance $h$. The unit cell contains a number of wells of depth $L$ acting as resonators. If the periodic structure is observed as a whole, there is a range of frequencies where it shows the so-called double-negative behavior: negative effective bulk modulus and negative effective mass. As a consequence, the lossless models predict that the metamaterial has extraordinary properties such as tunneling through narrow channels, control of the radiation field, perfect transmission through sharp corners and power splitting.

Two versions of the metamaterial were produced by 3D printing and used for measurements by Graciá-Salgado et al.. The scattering units were distributed in a hexagonal lattice with a lattice constant $a$ of $21 \mathrm{~mm}$. The measurements in Ref. [17] were made by insert- 


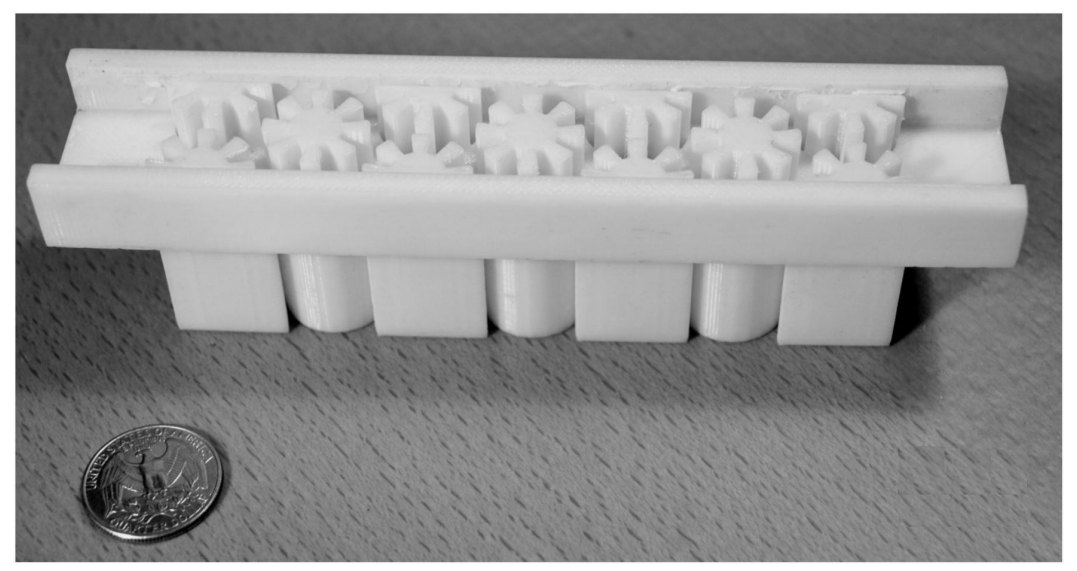

Fig. 2. Picture of one of the two metamaterial samples that were fabricated and measured by Graciá-Salgado et al, labelled Sample A. This sample is modeled in this paper using FEM and BEM with losses.

Table 1. Geometrical parameters of the two metamaterial samples used in experiments by Graciá-Salgado et al.. See Fig. 1 for a graphical description.

\begin{tabular}{ccccccc}
\hline Sample & $\begin{array}{c}R_{a} \\
(\mathrm{~mm})\end{array}$ & $\begin{array}{c}R_{b} \\
(\mathrm{~mm})\end{array}$ & $\begin{array}{c}\mathrm{h} \\
(\mathrm{mm})\end{array}$ & $\begin{array}{c}\mathrm{L} \\
(\mathrm{mm})\end{array}$ & $\begin{array}{c}\mathrm{a} \\
(\mathrm{mm})\end{array}$ & $\begin{array}{c}\alpha \\
(\mathrm{rad})\end{array}$ \\
\hline $\mathrm{A}$ & 4.6 & 9.2 & 9 & 31.5 & 21 & $\pi / 8$ \\
$\mathrm{~B}$ & 3.5 & 7 & 9 & 22.5 & 21 & $\pi / 8$ \\
\hline
\end{tabular}

ing the fabricated section of the periodic structure into a rectangular duct and exposing it to a wave propagating inside. In order to evaluate the transmittance, the sound pressure measured at the end of the duct was compared with an equivalent reading in a rectangular duct with no metamaterial. The duct is narrow enough to ensure a good approximation to a plane wave with no high-order propagating modes in the bandwidth of interest (1 to 5 $\mathrm{kHz}$ ). Two different samples of the metamaterial were fabricated with different geometrical parameters, one of them shown in Fig. 2. The dimensions are listed in Table 1. The samples are delimited by hard walls that are smooth and rigid, creating reflections and resembling a structure that extends infinitely in the direction normal to the wave on the lattice plane.

The measurement results did not confirm the expected double-negative behavior in any of the two samples. In the following sections, these results will be compared to models with losses. Only results for Sample A are presented in this paper.

\section{Description of the numerical implementations}

The two numerical implementations of acoustics with thermal and viscous losses are outlined in the following. A short description of each of them is followed by model details of the metamaterial test case. 


\subsection{BEM with losses}

The BEM implementation with losses is based on the Kirchhoff decomposition of the NavierStokes equations, ${ }^{6,7}$

$$
\begin{gathered}
\left(\Delta+k_{a}^{2}\right) p_{a}=0 \\
\left(\Delta+k_{h}^{2}\right) p_{h}=0 \\
\left(\Delta+k_{v}^{2}\right) \vec{v}_{v}=\overrightarrow{0}, \text { with } \Delta \cdot \vec{v}_{v}=0
\end{gathered}
$$

where the indexes $(a, h, v)$ represent the so-called acoustic, thermal and viscous modes, which can be treated independently in the acoustic domain and linked through the boundary conditions. The total pressure is the sum $p=p_{a}+p_{h}$ of the acoustic and thermal components (there is no pressure associated with the viscous mode), while the velocity has contributions from all three modes $\vec{v}=\vec{v}_{a}+\vec{v}_{h}+\vec{v}_{v}$. The wavenumbers $k_{a}, k_{h}$ and $k_{v}$ are based on the lossless wavenumber $k$ and physical properties of the fluid, such as the viscosity, bulk viscosity and thermal conductivity coefficients, air density, and specific heats. Eq.(1) is a wave equation, while Eqs. $(2,3)$ are diffusion equations, given the large imaginary part of $k_{h}$ and $k_{v}$. Eq. (3) is a vector equation and therefore it can be split into three components, giving a total of five equations with five unknowns.

The implementation in BEM is made by discretizing equations Eqs. $(1,2,3)$ independently and combining them into a single matrix equation using the boundary conditions. The matrix equation is solved for the acoustic pressure $p_{a}$ and subsequently other variables are obtained on the boundary. From the boundary values, any domain field point can be calculated. ${ }^{4,5}$ The implementation is based on the research software OpenBEM, which solves the Helmholtz wave equation using the direct collocation technique. ${ }^{19}$ Eqs. $(1,2,3)$ are formally equivalent to the Helmhotz wave equation.

In BEM, only the domain boundary is meshed, saving degrees of freedom as compared with other numerical methods like the FEM. However, the BEM coefficient matrices are frequency dependent and fully populated, which may counterbalance the mesh reduction when compared with other methods. In the case of BEM with viscous and thermal losses, three sets of coefficient matrices are used, corresponding to the three modes: acoustic, thermal and viscous. The thermal and viscous coefficient matrices are usually sparse due to the short reach of such effects, as compared with the overall dimensions of the setup.

\subsubsection{Description of the BEM metamaterial model}

The boundary mesh, shown in Fig. 3, has been created using the Gmsh grid generator. ${ }^{20}$ The metamaterial mesh has 6799 nodes and 13606 elements. The BEM implementation, and the OpenBEM software it is based on, are fully implemented in the Matlab programming language and no external solvers or preconditioners are used. The calculation of the coefficient matrices needs about one hour per frequency in a six-core $3,5 \mathrm{GHz}$ processor with 


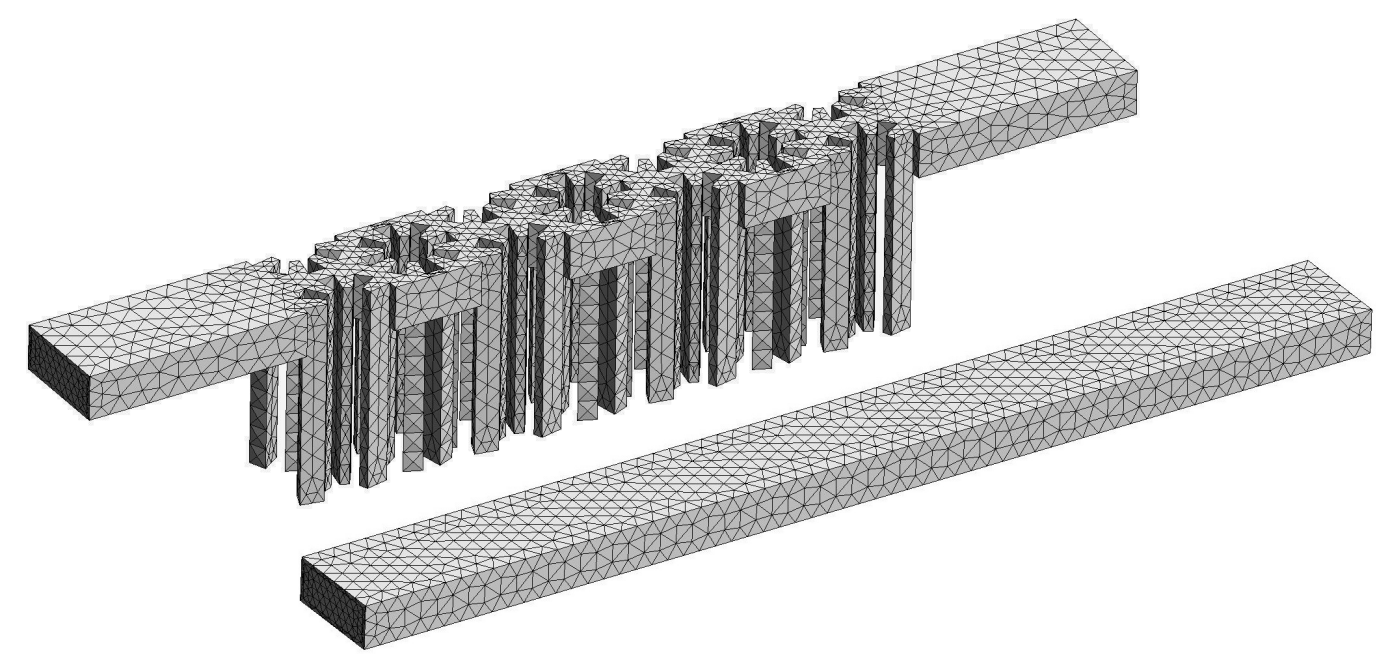

Fig. 3. Boundary mesh employed in the BEM model with losses. Upper, metamaterial sample; lower, empty duct. Linear three-node triangular elements are used.

$64 \mathrm{~Gb}$ of memory. The limiting factor is processor capacity, rather than memory. Solving the system takes six minutes per frequency. The size of the system to be solved is the same as the number of degrees of freedom of the boundary mesh. The calculated frequencies are spaced by $100 \mathrm{~Hz}$ from 1 to $5 \mathrm{kHz}$, giving forty-one frequencies. The sparsity of the thermal and viscous coefficient matrices is believed to enable some speed increase of the calculation, but it has not been directly exploited.

In parallel, an empty rectangular duct of the same dimensions has been calculated. The purpose of the empty duct is acting as a reference for the calculation of the metamaterial transmittance and reflectance. This reference could also be calculated analytically, but the numerical version serves as a test for the calculation issues described in the following.

The duct where the metamaterial is inserted and the empty duct used as a reference are assumed sufficiently long so as no reflections are created on any of the two terminations, emitting and receiving. In the FEM calculation described later, perfectly matched layers are used for this purpose. In the BEM code, the emitter and receiving lids are given an impedance of $\rho c$ instead, to make them anechoic to an incoming plane wave. One of the terminations acts as a plane piston with a normal velocity of $1 / \rho \mathrm{cm} / \mathrm{s}$ amplitude, so as to normalize the resulting plane wave to an amplitude of one.

The boundary conditions are set so as the mesh nodes on the rim of the lids can represent a sharp transition between the lid and the duct walls. This is done by splitting the columns in the acoustic coefficient matrix corresponding to the normal derivative of the pressure in the acoustic mode. ${ }^{21}$

In order to compare with the measurements in [17], transmitted power at the receiving end needs to be calculated. Pressure and particle velocity in the propagation direction (Xcoordinate, duct length direction) are calculated on ten field points situated on a plane that 
is normal to the propagation direction, thus allowing an estimation of the acoustic intensity and power. An equivalent calculation is made at the emitting side which is used to calculate the reflectance of the metamaterial. Measurement results are not available for the latter calculation, and it is not shown in this paper.

\subsection{FEM with losses}

Corresponding FEM simulations were carried out using the commercial software COMSOL. To accurately capture the effects of viscosity and thermal conduction for complex geometries like the metamaterial, the full linearised Navier-Stokes description is necessary. The equations solved are the momentum, continuity and energy equations,

$$
\begin{gathered}
i \omega \rho_{0} \mathbf{u}=\nabla \cdot\left(-p \mathbf{I}+\mu\left(\nabla \mathbf{u}+\nabla \mathbf{u}^{T}\right)-\left(\frac{2}{3} \mu-\mu_{B}\right)(\nabla \cdot \mathbf{u}) \mathbf{I}\right)+\mathbf{F} \\
i \omega \rho+\rho_{0} \nabla \cdot \mathbf{u}=0 \\
i \omega \rho_{0} C_{p} T=-\nabla \cdot(-k \nabla T)+i \omega \alpha_{0} T_{0} p \\
\rho=\rho_{0}\left(\beta_{T} p-\alpha_{0} T\right)
\end{gathered}
$$

The acoustic variables are; particle velocity $\mathbf{u}$, pressure $p$, temperature $T$. $\mathbf{F}$ is a volume force acting on the fluid. The parameters of air are expressed as: $\rho_{0}$ the equilibrium density, $T_{0}$ the equilibrium temperature, $\mu$ the dynamic velocity, $\mu_{B}$ the bulk viscosity, $C_{p}$ the heat capacity at constant pressure, $k$ thermal conductivity, $\alpha_{0}$ coefficient of thermal expansion and $\beta_{T}$ isothermal compressibility.

The above equation-set is solved using the regular FEM approach, transforming the equations into weak form. This results in a system of equations having pressure, particle velocity and temperature as variables. Five degrees of freedom are introduced per node, meaning that the system in general will be five times larger as compared to the lossless counterpart for the same mesh.

\subsubsection{Description of the FEM metamaterial model}

The model geometry is shown in Fig. 4, highlighting the Perfectly Matched Layers (PML) and the excitation. The models of the empty duct and the actual metamaterial are shown together, but the two geometries are solved separately, so that more elements in the metamaterial computation can be employed. Excitation of the waveguides is done though a body force with a small gap of air followed by a PML. This configuration was chosen to ensure full absorption at the inlet of waves reflected back from the metamaterial. Similarly, waves emerging at the other end of the metamaterial section are absorbed in the model with another PML. 


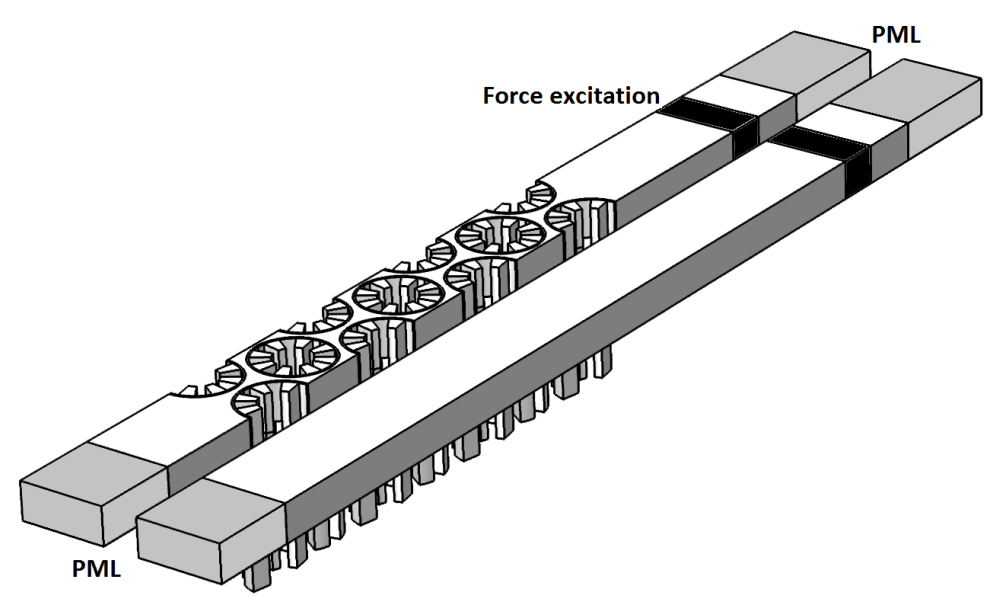

Fig. 4. Geometry of the FEM model with losses, including the metamaterial sample and the reference rectangular duct. The PML terminations and force excitations are marked in the drawings.

In order to capture the effects of viscosity and thermal conduction in FEM, the viscous and thermal boundary layers need to be meshed with mesh densities that are much higher than in the remaining domain regions. In complex models like the metamaterial sample modeled in this paper, this will mean a substantial increase of the number of degrees of freedom. The computer used for the FEM simulations has a six-core processor with $128 \mathrm{~GB}$ of memory. The presented results are computed with a memory load of approximately 100 GB. Computation of the full frequency range is carried out with a $25 \mathrm{~Hz}$ stepping, taking approximately 21 hours. The resulting system consists of 3.6 million degrees of freedom, solved with an iterative solver using direct preconditioners. ${ }^{22}$

Due to the usually larger system size of the viscothermal FEM formulation, special attention must be paid to the meshing of the domain, especially near wall regions where viscosity and thermal conduction become important. Boundary layer meshes for the entire geometry have been applied. Several configurations ranging from one to five element layers within the viscothermal boundary layers have been studied. These different computations only show insignificant changes in the computational result, indicating that the calculations can be limited to a very rough boundary layer mesh containing only one element within the boundary layer, thus reducing the overall computational load. Nevertheless, the presented FEM results correspond to simulations with the highest possible mesh density near boundaries.

\section{Results}

As previously mentioned, there are measurements available from Graciá-Salgado et al. of the transmittance (transmitted power/incident power) of the metamaterial. Fig. 5 shows these measurements together with the results from BEM and FEM with losses. The FEM calculation with no losses (also from Ref. [17]) is also shown. The lossless metamaterial 


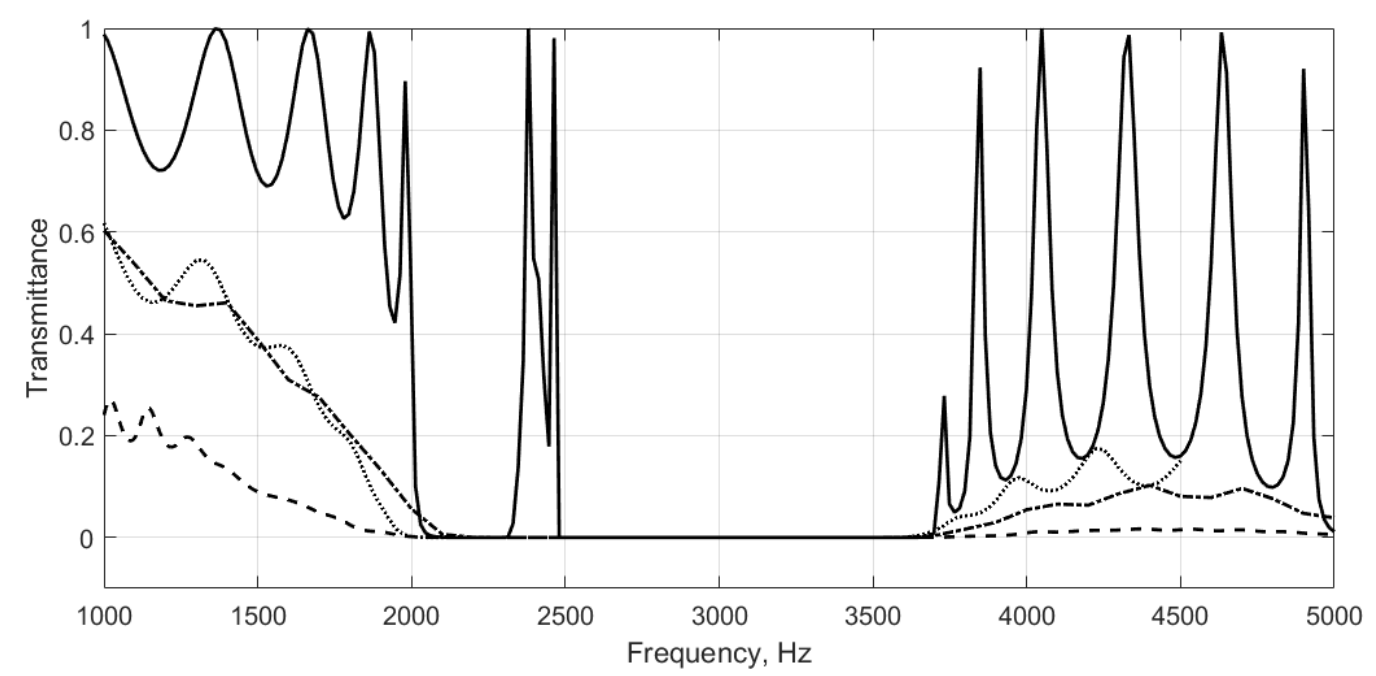

Fig. 5. Transmittance, obtained at the receiving end of the setup. Solid line, FEM model without losses; dotted line, FEM model with losses; dash-dotted line, BEM model with losses; dashed line, measurements by Graciá-Salgado et al..

exhibits negative density and bulk modulus (double-negative behavior) within a narrow band close to $2,5 \mathrm{kHz}$, which disappears when losses are present. This is an important outcome that may have an impact in metamaterial design.

In this paper we are concerned however with the performance of the numerical methods. The BEM and the FEM calculations with losses appear to be rather close to each other, and away from the measurement results. Only at high frequencies this picture is less clear, possibly a consequence of the BEM mesh density becoming insufficient. Indeed all three results with losses (BEM, FEM and measurements) are difficult to obtain and subject to errors, so no clear-cut conclusion can be drawn. In FEM, it has not been possible to increase significantly the mesh at the boundary layers in order to study its effect. In BEM, a mesh with less nodes than the one employed in this paper showed similar results. Also in BEM, a drastic increase in mesh density would also make the calculation unaffordable. However, to some extent the FEM and BEM results validate each other.

Using the numerical calculations, it is possible to examine the behavior of the structure in a detail that is not reachable to measurements. As an example, Fig. 6 shows the pressures along the propagation direction on the boundary of the metamaterial within the doublenegative band, calculated using BEM. Note that the BEM predicts the double-negative behavior at a frequency of around $2600 \mathrm{~Hz}$, and this value is used in Fig. 6. The FEM calculation with no losses in Fig. 5 shows a double negative band at 2400-2500 Hz.

If the duct was plain and empty, the boundary conditions would produce a plane progressive wave of amplitude 1, which is marked in Fig. 6 as thin solid lines. The amplitudes in the resonator units are much larger, and in the simulation with no losses they combine in such a way as to produce the extraordinary behavior described by Graciá-Salgado et al.. The same figure shows the effect of losses, which prevent wave propagation to the receiving 

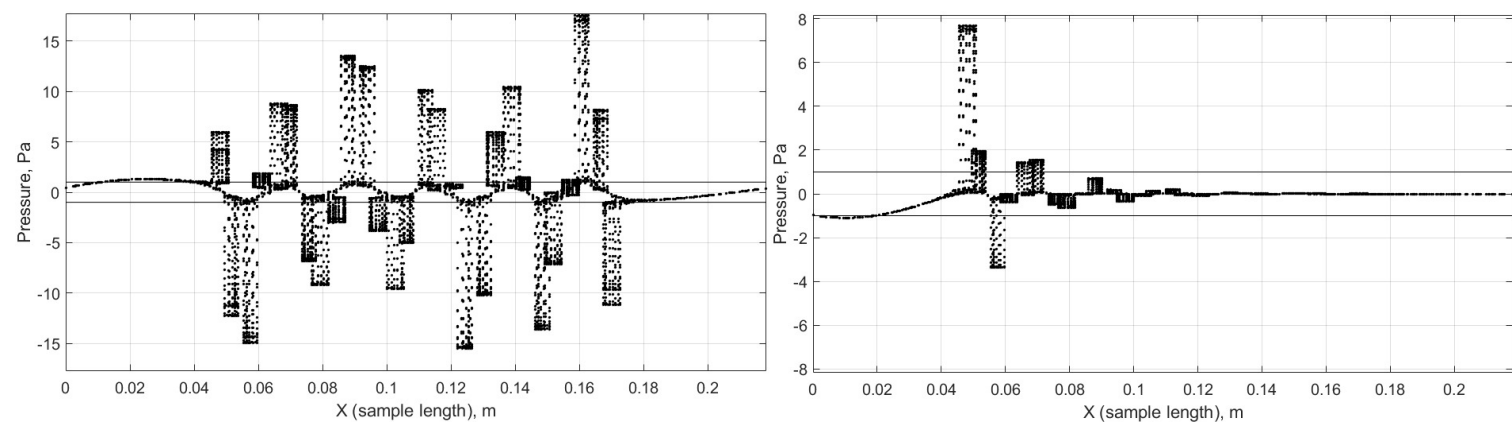

Fig. 6. Sound pressure at a given instant of time on the surface mesh, calculated with BEM. The abscissas are the positions of the nodes in the propagation direction. The frequency is $2600 \mathrm{~Hz}$, which is within the double-negative band predicted by BEM. Left, calculation with no losses; right, calculation with viscous and thermal losses.

end and destroy the interplay between metamaterial units.

\section{Conclusions}

A metamaterial test case from the literature has been modeled using BEM and FEM with losses. The simulations confirm the conclusion from existing measurement results: the double-negative behavior associated with the metamaterial's extraordinary properties is prevented by viscous and thermal losses. This conclusion applies in principle to this particular metamaterial, but may serve as a warning when estimating the behavior of similar structures.

The test case is a challenging calculation both for BEM and FEM with losses. Numerical transmittance results with losses using FEM and BEM are not far from each other, and both predict less losses than the measurements. This can serve, to some extent, as a verification of the numerical implementations with losses. However, it should be kept in mind that measurements are also challenging and subject to deviations. Further refinement of the numerical models would be necessary to obtain a better estimation, but this would lead to a drastic increase of the computational burden, which is not bearable with the computers used in this work. A possible way forward would be the creation of more efficient implementations of acoustic losses.

\section{Acknowledgments}

The authors wish to thank Mads J. Herring Jensen, from the company COMSOL, for his support in setting up the FEM model of the metamaterial.

J. Sánchez-Dehesa acknowledges the support by the Spanish Ministerio de Economía y Competitividad, and the European Union Fondo Europeo de Desarrollo Regional (FEDER) through Project No. TEC2014-53088-C3-1-R. 


\section{References}

1. R. V. Craster and S. Guenneau, Eds., Acoustic Metamaterials. Negative Refraction, Imaging, Lensing and Cloaking (Springer, London, 2013).

2. S. A. Cummer, J. Christensen, A. Alù, Controlling sound with acoustic metamaterials. Nature Reviews Materials art. no. 1601, pp. 1-13 (2016).

3. P. M. Morse and K. U. Ingard, Theoretical Acoustics (McGraw-Hill, Princeton, 1968).

4. V. Cutanda Henríquez and P. M. Juhl, An axisymmetric boundary element formulation of sound wave propagation in fluids including viscous and thermal losses. J. Acoust. Soc. Am., 134(5), 3409-3418, (2013).

5. V. Cutanda Henríquez and P. M. Juhl, Implementation of an Acoustic 3D BEM with ViscoThermal Losses, Internoise 2013, 15-18 Sept. 2013, Innsbruck, Austria.

6. A. D. Pierce, Acoustics. An introduction to its physical principles and applications (Ch. 10) (McGraw Hill, New York, 1981).

7. M. Bruneau, Ph. Herzog, J. Kergomard and J. D. Polack, General formulation of the dispersion equation in bounded visco-thermal fluid, and application to some simple geometries, Wave Motion 11, 441-451 (1989).

8. V. Cutanda Henríquez, P. M. Juhl, Modelling measurement microphones using BEM with viscothermal losses. Joint Baltic-Nordic Acoustics Meeting, 18-20 June 2012, Odense, Denmark.

9. V. Cutanda Henríquez, S. Barrera-Figueroa, A. Torras Rosell, P. M. Juhl, Study of the acoustical properties of a condenser microphone under an obliquely incident plane wave using a fully coupled three-dimensional numerical model. Internoise 2015, 9-12 Aug. 2015, San Francisco (USA).

10. R. Bossart, N. Joly and M. Bruneau. Methodes de modelisation numerique des champs acoustiques en fluide thermovisqueux. Actes du $6^{e}$ Congrès Franais d'Acoustique, Lille, France (2002), pp. 411-414.

11. M. Malinen, M. Lyly, P. Råback, A. Kärkkäinen and L. Kärkkäinen, A finite element method for the modeling of thermo-viscous effects in acoustics. Proc. $4^{\text {th }}$ European Congress Computational Methods in Applied Sciences and Engineering ECCOMAS, Jyväskylä, Finland (2004).

12. N. Joly, Coupled equations for particle velocity and temperature variation as the fundamental formulation of linear acoustics in thermo-viscous fluids at rest. Acta Acust. Acust. 92, 202209, (2006).

13. R. Kampinga, Performance of several viscothermal acoustic finite elements. Acta Acust. Acust. 96, 115124, (2010).

14. COMSOL Multiphysics Reference Manual, version 5.2 (2015).

15. R. Kampinga, An efficient finite element model for viscothermal acoustics, Acta Acust. Acust. 97, 618631, (2011).

16. W. M. Beltman. Viscothermal wave propagation including acousto-elastic interaction. Part I: Theory and Part II: Applications, J. Sound Vib. 227 (3), 555-586 (part I) and 587-609 (part II), (1999).

17. R. Graciá-Salgado, V. M. García-Chocano, D. Torrent and J. Sánchez-Dehesa, Negative mass density and $\rho$-near-zero quasi-two-dimensional metamaterials: Design and applications, Phys. Rev. B 88, 224305 (2013).

18. D. Homentcovschi and R. N. Miles, An analytical-numerical method for determining the mechanical response of a condenser microphone, J. Acoust. Soc. Amer. 130(6), 36983705, (2011).

19. V. Cutanda Henríquez and P. M. Juhl, OpenBEM - An open source Boundary Element Method software in Acoustics, Internoise 2010, 13-16 Jun. 2010, Lisbon, Portugal.

20. C. Geuzaine and J.-F. Remacle. Gmsh: a three-dimensional finite element mesh generator with built-in pre- and post-processing facilities. International Journal for Numerical Methods in Engineering 79(11), pp. 1309-1331, 2009. 
21. P. M. Juhl, The boundary Element Method for Sound Field Calculations, Ph.D. thesis, Report No. 55, Technical University of Denmark, 1993.

22. COMSOL Multiphysics Acoustics Module User's Guide, version 5.2 (2015) 